\title{
De Winter ECG Pattern Can Be A Temporary ECG Phenomenon Related To Severe Stenosis of The Left Circumflex Coronary Artery: A Case Report
}

Jian Wang ( $\sim_{\text {jian19880907@126.com ) }}$

Binzhou Medical University Hospital

Faming Ding

Binzhou Medical University Hospital

Jingsen Li

Binzhou Medical University Hospital

Huipu Xu

Binzhou Medical University Hospital

\section{Case report}

Keywords: De Winter ECG pattern, ST elevation equivalent, left circumflex coronary artery

Posted Date: August 18th, 2021

DOI: https://doi.org/10.21203/rs.3.rs-789601/v1

License: (c) (i) This work is licensed under a Creative Commons Attribution 4.0 International License. Read Full License 


\section{Abstract}

The de Winter ECG pattern consisting of ST-segment depression and tall symmetrical T waves on ECG, known as an ST elevation equivalent, accounts for approximately $2 \%$ of patients with occlusion of the proximal left anterior descending coronary artery (LAD). These patterns are considered static and persistent and are, on average, recorded 1.5 hours after onset. Here, we describe a case of the de Winter ECG pattern as a temporary ECG phenomenon associated with left circumflex coronary artery (LCX) stenosis.

\section{Introduction}

De Winter et al [1] first described the de Winter ECG pattern in the New England Journal of Medicine in 2008. The de Winter ECG pattern, rather than ST-segment elevation, shows a 1-3 mm upsloping ST segment depression at the $\mathrm{J}$ point in precordial leads (V1-V6) followed by tall, positive symmetrical $\mathrm{T}$ waves and ST elevation of 1-2 mm in lead aVR, which have severe stenosis of the LAD, causing extensive anterior myocardial infarction. This ECG pattern continues until revascularization of the occluded vessel occurs $[2,3,4]$. A recent systematic review suggests that the positive predictive value of the de Winter pattern is close to $100 \%$ for acute coronary occlusion [5]. Therefore, recognition of this ECG pattern for physicians is of vital importance, and emergency reperfusion therapy must be performed as the ST elevation myocardial infarction (STEMI) equivalent.

\section{Case Report}

A 65-year-old male, who was a current smoker with hypertension, was admitted to our hospital with chest pain radiating to the jaw for 6 months and aggravation for 1 month. Chest pain induced by activity persisted for approximately 10-20 minutes each time, and was gradually alleviated after rest. Physical examination revealed an anxious appearance. His pulse rate was 54 beats/min, his blood pressure was $121 / 73 \mathrm{mmHg}$, and his respiratory rate was 16 breaths/min. The initial ECG showed slight ST-segment elevation in I, aVL with T-wave inversion in the inferior leads (Fig. 1A). The cardiac troponin I level was 0.07 (normal value $0-0.09 \mathrm{ng} / \mathrm{ml}$ ) and all other basic laboratory tests were within normal limits. An echocardiogram showed that ventricular diastolic function had decreased. He was treated with aspirin, ticagrelor, enoxaparin sodium, amlodipine, atorvastatin and isosorbide mononitrate. One day after admission, the patient presented with sudden chest pain radiating to the jaws with diaphoresis while he was defecating. An ECG was performed, showing upsloping ST-segment depression up to $6 \mathrm{~mm}$ at the $\mathrm{J}$ point from $\mathrm{V} 1$ to $\mathrm{V} 6, \mathrm{I}$, aVL; tall symmetrical $\mathrm{T}$ waves in leads $\mathrm{V} 2-\mathrm{V} 5$ together with a $1 \mathrm{~mm} \mathrm{~J}$ point elevation in the lead aVR; and elevation at the $\mathrm{J}$ point in the inferior leads with a heart rate of 34 times/min (Fig. 1B). At the same time, after performing a long II lead ECG, cardiac arrhythmia was revealed, including sinus bradycardia, atrioventricular (AV) junctional escape beats and AV junctional rhythm (Fig. 2). After the administration of nitroglycerin $(5 \mathrm{mg})$ three minutes later, the patient became symptom-free, and repeated ECG showed that the pattern had returned to its former shape. We recognized the de Winter ECG pattern, which should be treated as the ST-elevation equivalent. However, 
given the stable hemodynamic status, and relief of chest pain after treatment, the cardiac catheterization laboratory was not activated immediately.

Twenty hours later, a coronary arteriogram was performed, confirming total occlusion of the proximal right coronary artery (RCA) with collateral circulation (Fig. 3) from the left coronary artery and no significant lesions at the LAD or the left main artery; the LCX was almost completely occluded at its middle portion, followed by insertion of a drug-eluting stent (Fig. 3). A few days later, the patient was discharged without any sign of complications.

\section{Discussion}

Our case verifies that the de Winter ECG pattern is not exclusively associated with occlusion of the proximal LAD and may be a transient ECG phenomenon.

Initially, the de Winter ECG pattern was considered static and persistent and, on average, recorded 1.5 hours after onset [1]. Recent case reports demonstrate that the de Winter ECG pattern can evolve to a STEMI ECG pattern within hours of presentation [2, 3], which may be due to a lack of recognition [4]. The difference in our case is that after administration of nitroglycerin, the de Winter ECG pattern returned to its former shape which was inconsistent with previous reports. We speculate that it may be related to recanalization after embolization.

In the present case, it is worth noting that the ECG pattern recorded in this patient showed elevation at the $J$ point in the inferior leads with bradyarrhythmia which included sinus bradycardia, AV junctional escape beats and $A V$ junctional rhythm. Why did they occur? It is well-known that the blood supply to the sinoatrial (SA) node is also most often derived from the RCA ( $70 \%$ of the time). However, in $25 \%$ of normal hearts, the SA nodal artery arises from the LCX, and in 5\% of cases, both the RCA and the LCX contribute to this vessel. The coronary arteriogram revealed occlusion of the proximal RCA with collateral circulation from the left coronary artery, and the LCX was almost completely occluded. We speculate that when the LCX was occluded, blood flow to the RCA and SA nodal artery was decreased, which led to elevation at the $J$ point in the inferior leads and bradyarrhythmia. In addition, we should take into consideration that the occurrence of a junctional rhythm may cause a misinterpretation of QRS morphology. The de Winter ECG pattern is caused by occlusion of the LAD or its branches or RCA $[6,7,8]$, and no prior description of this pattern associated with occlusion of the LCX has been reported. We considered some of the reasons for this abnormal ECG change related to severe LCX stenosis as follows: individual differences in coronary artery anatomy and recruitment of collateral channels [6,9]. Physicians should recognize this pattern and need to perform emergent angiography and reperfusion therapy.

\section{Declarations}

\section{Ethics approval and consent to participate}


This case report has been conducted according to the standards of the Declaration of Helsinki. The written content to publish was obtained from the patient.

\section{Consent for publication}

Content to publish was obtained from the patient.

\section{Availability of data and material}

All data is available and can be provided if requested.

\section{Competing of interest}

The authors declare no potential conflicts of interest.

\section{Funding}

No.

\section{Authors' contributions}

JW performed the collection of data and wrote the manuscript. FD performed the literature search. JL and $\mathrm{HX}$ guaranteed of the integrity of the entire study. All authors have read and approved the manuscript reviewed the literature.

\section{Acknowledgements}

The authors would like to acknowledge Joan McDermott for her help with polishing.

\section{References}

1. de Winter RJ, Verouden NJ, Wellens HJ, Wilde AA. A new ECG sign of proximal LAD occlusion. N Engl J Med. 2008;359:2071-3.

2. Wei-Wei X, Liang L, Mei-Juan J de. Winter Electrocardiogram Pattern-An Unusual ST-Segment Elevation Myocardial Infarction Equivalent Pattern. JAMA Intern Med. 2019;179:1575-7.

3. Goebel M, Bledsoe J, Orford JL, Mattu A, Brady WJ. A new ST-segment elevation myocardial infarction equivalent pattern? Prominent T wave and J-point depression in the precordial leads associated with ST-segment elevation in lead aVr. Am J Emerg Med. 2014;32:287.e5-8.

4. Xu J, Wang A, Liu L, Chen Z. The de winter electrocardiogram pattern is a transient electrocardiographic phenomenon that presents at the early stage of ST-segment elevation myocardial infarction. Clin Cardiol. 2018;41:1177-84.

5. Morris NP, Body R. The De Winter ECG pattern: morphology and accuracy for diagnosing acute coronary occlusion: systematic review. Eur J Emerg Med. 2017;24:236-42. 
6. Montero Cabezas JM, Karalis I, Schalij MJ. De Winter Electrocardiographic Pattern Related with a Non-Left Anterior Descending Coronary Artery Occlusion. Ann Noninvasive Electrocardiol. 2016;21:526-8.

7. Karna S, Chourasiya M, Chaudhari T, Bakrenia S, Patel U. De Winter sign in inferior leads: A rare presentation. Heart Views. 2019;20:25-7.

8. Xu W, Zou H, Huang S. Junctional ST-depression and tall symmetrical T-waves with an obtuse marginal artery occlusion: A case report. J Electrocardiol. 2019;54:40-2.

9. Sunbul M, Erdogan O, Yesildag O, Mutlu B. De Winter sign in a patient with left main coronary artery occlusion. Postepy Kardiol Interwencyjnej. 2015;11:239-40.

\section{Figures}

A

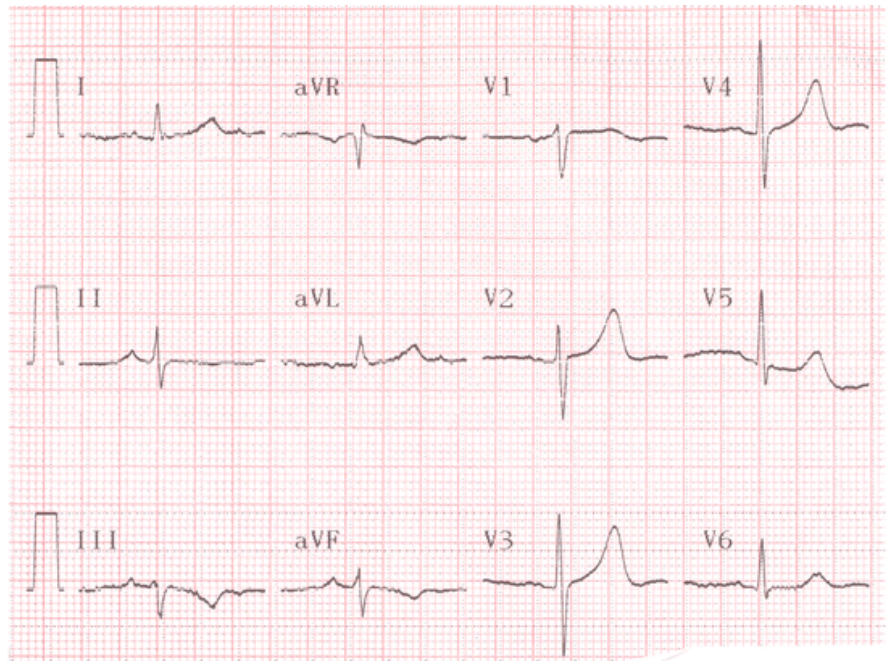

$\mathrm{B}$

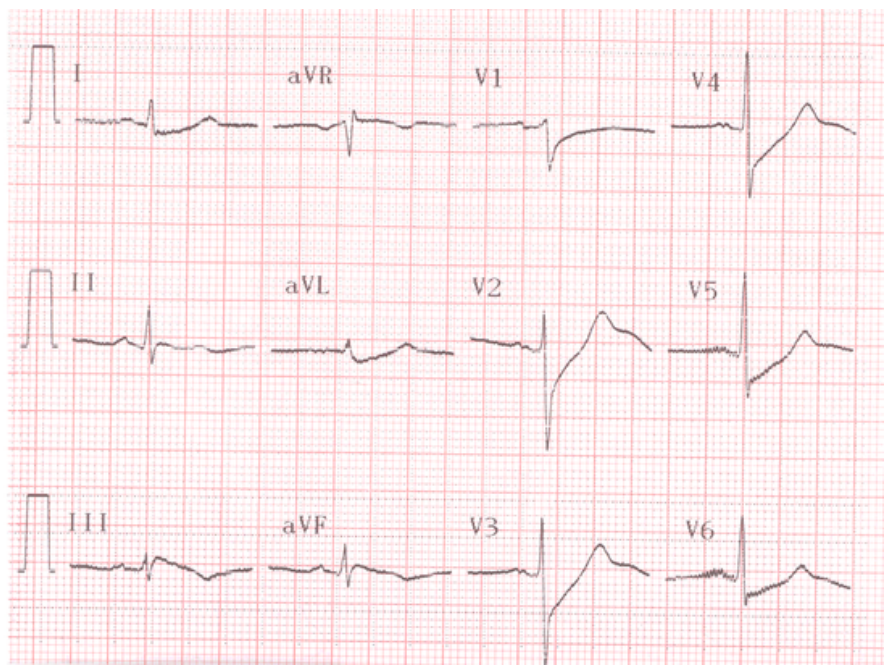

\section{Figure 1}

Twelve-lead ECG of the patient. (A) Initial ECG showed slight ST-segment elevation in I, aVL with T-wave inversions in the inferior leads. (B) When he experienced chest pain, ECG was performed, showing upsloping ST-segment depression at the J point from V1 to V6, I, aVL; tall symmetrical T waves in leads $\mathrm{V} 2-\mathrm{V} 5$ together with a $\mathrm{J}$ point elevation in the lead aVR; and elevation at the $\mathrm{J}$ point in the inferior leads with a heart rate of 34 times/min. 


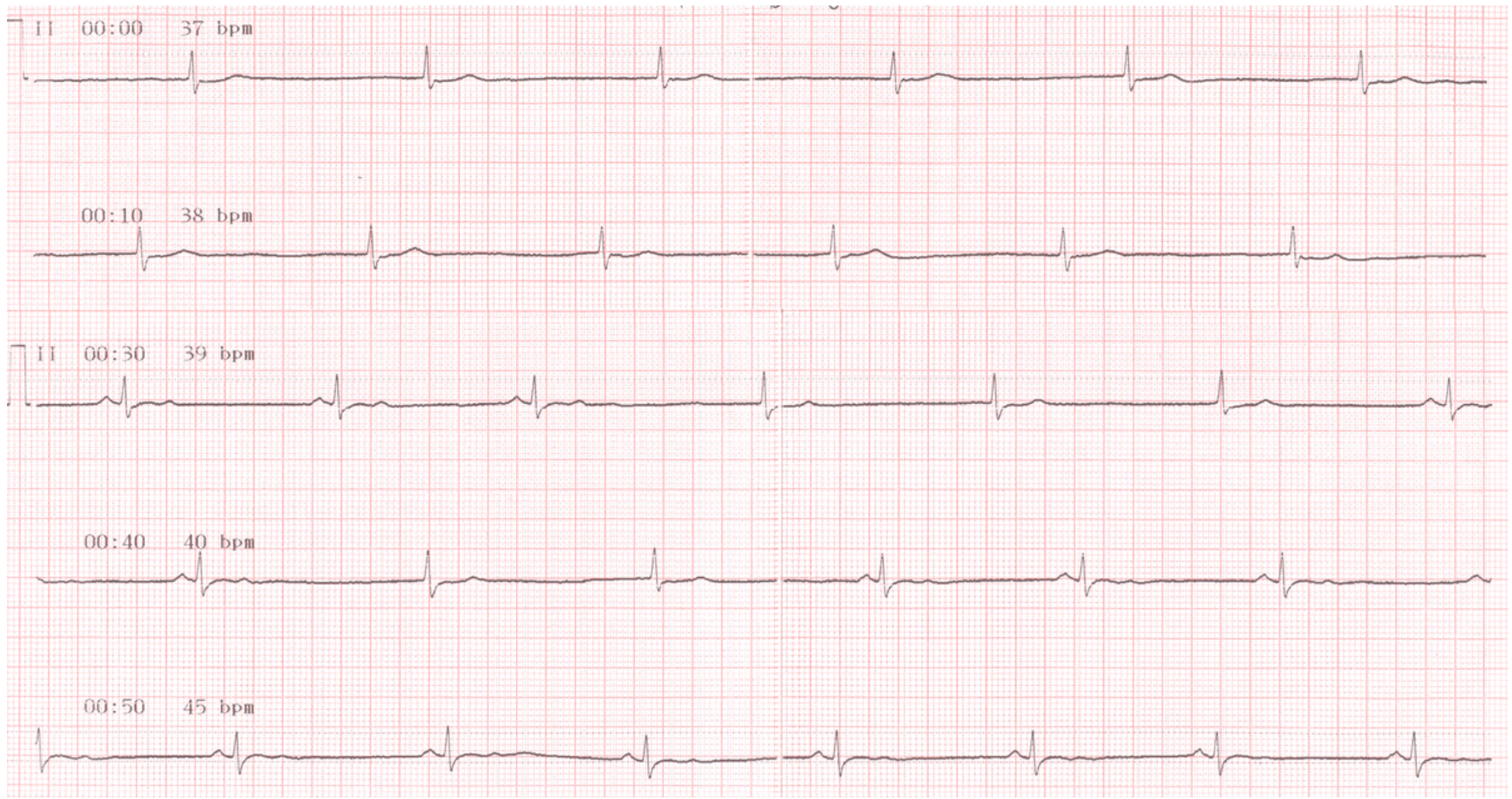

Figure 2

II lead ECG of the patient. After performing a long II lead ECG, cardiac arrhythmia was revealed, including sinus bradycardia, atrioventricular (AV) junctional escape beats and $\mathrm{AV}$ junctional rhythm. 


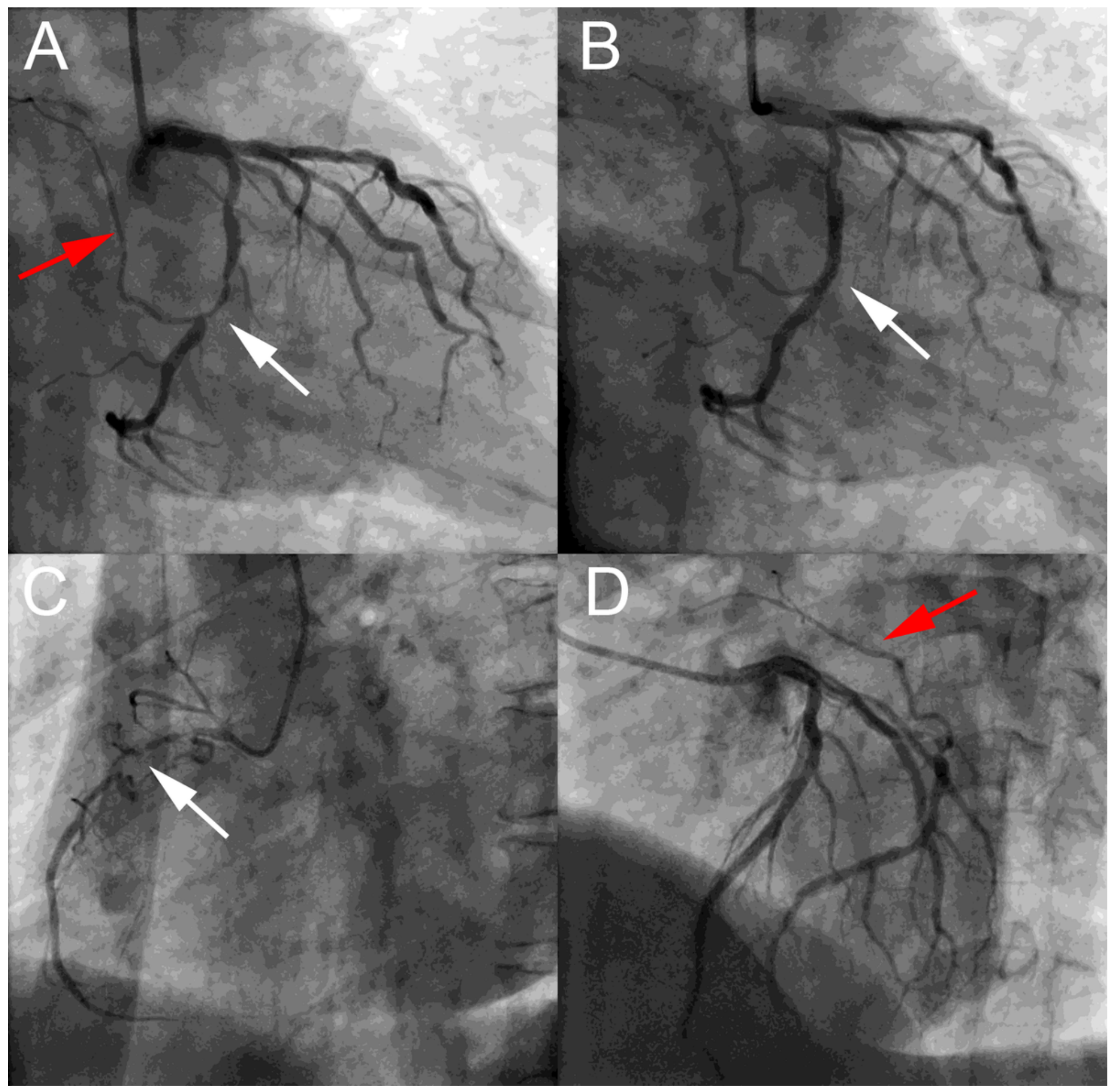

\section{Figure 3}

Coronary angiography. Coronary angiogram showed that the left circumflex coronary artery (LCX) was almost completely occluded at its middle portion (A, white arrow), followed by insertion of a drug-eluting stent ( $B$, white arrow). Proximal right coronary artery occlusion (RCX) was observed (C, white arrow) with collateral circulation from the left coronary artery $(A, D$, red arrow). 